\title{
Nanocrystals-Related Synthesis, Assembly, and Energy Applications
}

\author{
Quanqin Dai, ${ }^{1}$ Bo Zou, ${ }^{2}$ William W. Yu, ${ }^{3}$ Jaetae Seo, ${ }^{4}$ and Michael Z. Hu \\ ${ }^{1}$ Oak Ridge National Laboratory, Oak Ridge, TN 37831, USA \\ ${ }^{2}$ State Key Laboratory of Superhard Materials, Jilin University, Changchun 130012, China \\ ${ }^{3}$ Center for Biological and Environmental Nanotechnology, Rice University, Houston, TX 77005, USA \\ ${ }^{4}$ Department of Physics, Hampton University, Hampton, VA 23668, USA \\ Correspondence should be addressed to Quanqin Dai, connectdai@hotmail.com
}

Received 10 December 2010; Accepted 10 December 2010

Copyright (c) 2011 Quanqin Dai et al. This is an open access article distributed under the Creative Commons Attribution License, which permits unrestricted use, distribution, and reproduction in any medium, provided the original work is properly cited.

Fundamental material properties have been dramatically altered in the nanoscale regime because of quantum confinement effect. The unique size-tunable functionalities of nanomaterials make them involved in an extensive variety of energy applications, such as light-emitting diodes and solar cells. These applications have been demonstrated to cut energy consumption. In response to the ever-growing energy demands as well as the concerns of global warming, researchers are actively placing their enormous emphasis on the exploration of energy savings. During this exploration, the primary stage requires the design of appropriate strategies for the synthesis of high-quality nanocrystals in terms of size uniformity and superior optical/electronic properties. Especially, there is a need to seek green-chemistry approaches for the synthesis of environmentally benign and user-friendly nanocrystals. Another recent area of focus is the use of individual nanocrystals as building blocks for self-assembly, providing new opportunities to improve the nanocrystal performance.

Therefore, we organize the current special issue for Journal of Nanomaterials to provide the authors with a platform and readers with the latest achievements of nanocrystalsrelated synthesis, assembly, and energy applications. This special issue focuses on the fundamental science and technical applications of nanocrystals, covering the nanocrystalsrelated research area of synthesis, characterization, properties, assembly, films, processing, modeling, and devices for energy applications.

The articles related to synthesis, characterization and properties in this special issue include studies of the mechanisms and growth of flexible $\mathrm{ZnO}$ nanostructure arrays, facile synthesis of monodisperse $\mathrm{ZnO}$ nanocrystals, synthesis of vertically aligned dense $\mathrm{ZnO}$ nanowires, $\mathrm{CdS}$ nanotubes and Y-branched nanochannels fabricated by a double diffusion route, hydrothermal synthesis and characterization of singlecrystalline $\alpha-\mathrm{Fe}_{2} \mathrm{O}_{3}$ nanocubes, a user-friendly method for synthesizing high-quality $\mathrm{NaYF}_{4}: \mathrm{Yb}, \mathrm{Er}(\mathrm{Tm})$ nanocrystals, a rational self-sacrificing template route to $\mathrm{LiMn}_{2} \mathrm{O}_{4}$ nanotubes and nanowires, synthesis of bismuth ferrite nanoparticles via a wet-chemical route at low temperature, synthesis of Ag nanostructures by photochemical reduction using citrate-capped Pt seeds, study of the P-type doping properties of $\mathrm{ZnS}$ nanocrystals, structural and mechanical properties of $\mathrm{CrNx}$ coatings deposited by medium-frequency magnetron sputtering with and without ion source assistance, optical properties of linoleic acid protected gold nanoparticles, and so on.

Authors also contributed papers to the areas of assembly and films, such as ordered self-assembly of nanoPd-Ga/PMMA by ultrasonic processing, self-assembly of monodisperse spherical $\mathrm{TiO}_{2}$ nanocrystals, linear assembles of BN nanosheets, controllable assembly of hydrophobic superparamagnetic iron oxide nanoparticles with mPEGPLA copolymers, polythene films with doped and undoped $\mathrm{TiO}_{2}$ nanoparticles, and the influence of lithium on nanosized films of $\mathrm{Fe}_{2} \mathrm{O}_{3}$.

The research topic of processing and modeling contains articles on electronic-excitation-induced processing in $\mathrm{GaSb}$ compound nanoparticles, and constitutive rheological modeling of flow serration behavior in metallic glasses.

In the applications of nanocrystals, authors report CdSe quantum dot-sensitized mesoporous $\mathrm{TiO}_{2}$ solar cells with 
$\mathrm{CuSCN}$ as solid-state electrolyte, fluoride nanoscintillators, and electrodeposition of nanometer-sized ferric oxide materials in colloidal templates for conversion of light to chemical energy.

\section{Acknowledgments}

The editors would like to thank the authors for their contributions to this special issue, the reviewers for their time and dedication, and Rasha Magdy (Publishing Editor) and Mostafa Salem (Editorial Staff) for their support and assistance.

Quanqin Dai Bo Zou

William W. Yu

Jaetae Seo

Michael Z. Hu 

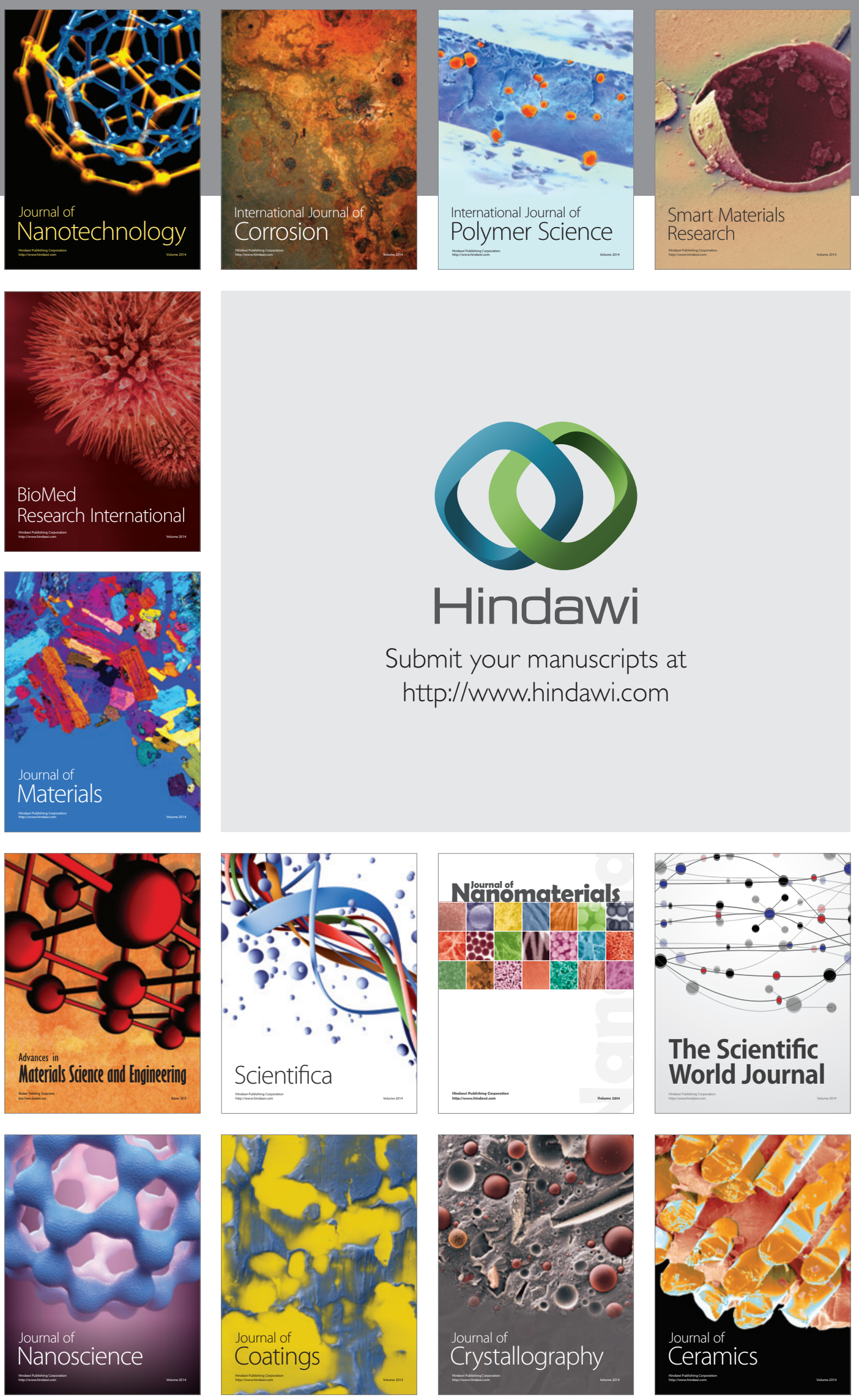

The Scientific World Journal

Submit your manuscripts at

http://www.hindawi.com

\section{World Journal}

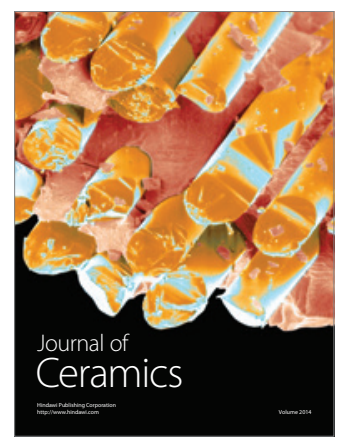

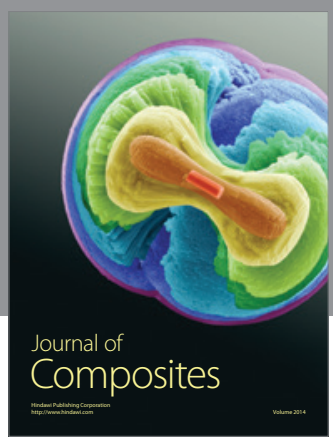
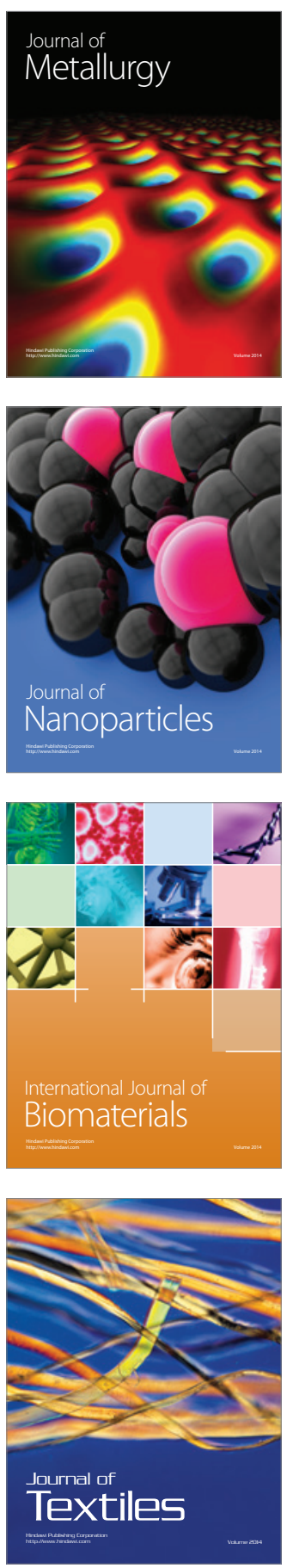